\title{
ERRATUM
}

\section{Erratum to: Higher-Order Averaging, Formal Series and Numerical Integration II: The Quasi-Periodic Case}

\author{
P. Chartier ${ }^{1}$ - A. Murua ${ }^{2}$ - J. M. Sanz-Serna ${ }^{3}$
}

Published online: 23 March 2016

(C) SFoCM 2016

\section{Erratum to: Found Comput Math (2012) 12:471-508 DOI 10.1007/s10208-012-9118-8}

After formula (22), the tree $u$ must have the label $\mathbf{l}$ at the root and the label $\mathbf{k}$ at the leaf. Furthermore, the condition $\mathbf{k} \neq \mathbf{0}$ must be imposed.

The formula at the second bullet point after (56) must be changed to $f_{\mathbf{k}_{1} \cdots \mathbf{k}_{r}}(y):=$ $\partial_{y} f_{\mathbf{k}_{2} \cdots \mathbf{k}_{r}}(y) f_{\mathbf{k}_{1}}(y)$.

In the unnumbered displayed formula before (61), the left-hand side must be $H(y, \theta)$ rather than $\epsilon H(y, \theta)$. In formula (61), the leftt-hand side must be $\epsilon \bar{H}$ rather than $\bar{H}$.

The online version of the original article can be found under doi:10.1007/s10208-012-9118-8.

J. M. Sanz-Serna

jmsanzserna@gmail.com

P. Chartier

Philippe.Chartier@inria.fr

A. Murua

Ander.Murua@ehu.es

1 INRIA Rennes and ENS Cachan Bretagne, Campus Ker-Lann, av. Robert Schumann, 35170 Bruz, France

2 Konputazio Zientziak eta A. A. Saila, Informatika Fakultatea, UPV/EHU, 20018 Donostia-San Sebastián, Spain

3 Departamento de Matemáticas, Universidad Carlos III de Madrid, E-28911 Leganés (Madrid), Spain 
The unnumbered displayed formula before (64) may be simplified to read:

$$
\begin{aligned}
\frac{\mathrm{d}}{\mathrm{d} t}= & \varepsilon \sum_{\mathbf{k}} \bar{\beta}_{\mathbf{k}} f_{\mathbf{k}}+\varepsilon^{2} \sum_{\mathbf{k}>\mathbf{l}} \bar{\beta}_{\mathbf{l k}}\left[f_{\mathbf{l}}, f_{\mathbf{k}}\right] \\
& +\varepsilon^{3}\left(\sum_{\mathbf{k} \neq \mathbf{l}} \bar{\beta}_{\mathbf{l l k}}\left[f_{\mathbf{l}},\left[f_{\mathbf{l}}, f_{\mathbf{k}}\right]\right]+\sum_{\mathbf{l}>\mathbf{k}<\mathbf{m}, \mathbf{m} \neq \mathbf{l}} \bar{\beta}_{\mathbf{m l k}}\left[f_{\mathbf{m}},\left[f_{\mathbf{l}}, f_{\mathbf{k}}\right]\right]\right) \\
& +\mathcal{O}\left(\varepsilon^{4}\right),
\end{aligned}
$$

where $<$ is some total ordering in the set of multi-indices $\mathbb{Z}^{d}$, such that $\mathbf{k}>\mathbf{0}$ for $\mathbf{k} \neq \mathbf{0}$. Formula (64) is wrong and must be replaced by

$$
\frac{\mathrm{d}}{\mathrm{d} t} Y=\varepsilon f_{0}+\varepsilon^{2} F_{2}+\varepsilon^{3} F_{3}+\mathcal{O}\left(\varepsilon^{4}\right)
$$

where, with $<$ as before,

$$
\begin{aligned}
& F_{2}=\sum_{\mathbf{k}>-\mathbf{k}} \frac{i}{\mathbf{k} \cdot \omega}\left(\left[f_{\mathbf{k}}-f_{-\mathbf{k}}, f_{\mathbf{0}}\right]+\left[f_{-\mathbf{k}}, f_{\mathbf{k}}\right]\right), \\
& F_{3}=\sum_{\mathbf{k} \neq \mathbf{0}} \frac{1}{(\mathbf{k} \cdot \omega)^{2}}\left(\left[f_{\mathbf{0}},\left[f_{\mathbf{0}}, f_{\mathbf{k}}\right]\right]+\left[f_{\mathbf{k}},\left[f_{\mathbf{k}}, f_{-\mathbf{k}}\right]\right]\right. \\
& \left.-\frac{1}{2}\left[f_{\mathbf{k}},\left[f_{\mathbf{k}}, f_{-2 \mathbf{k}}\right]\right]+\left[f_{-\mathbf{k}},\left[f_{\mathbf{k}}, f_{\mathbf{0}}\right]\right]\right) \\
& +\sum_{\mathbf{0} \neq \mathbf{m} \neq-\mathbf{l} \neq \mathbf{0}} \frac{-1}{(\mathbf{l} \cdot \omega)((\mathbf{m}+\mathbf{l}) \cdot \omega)}\left[f_{\mathbf{m}},\left[f_{\mathbf{l}}, f_{\mathbf{0}}\right]\right] \\
& +\sum_{-\mathbf{l}>\mathbf{k}<\mathbf{l}, \mathbf{k} \neq \mathbf{0}} \frac{1}{(\mathbf{k} \cdot \omega)(\mathbf{l} \cdot \omega)}\left[f_{-\mathbf{l}},\left[f_{\mathbf{l}}, f_{\mathbf{k}}\right]\right] \\
& +\sum_{\substack{\mathbf{m}>\mathbf{k}<-\mathbf{k} \\
\mathbf{m}+\mathbf{k} \neq \mathbf{0}}} \frac{-1}{(\mathbf{k} \cdot \omega)(\mathbf{m} \cdot \omega)}\left[f_{\mathbf{m}},\left[f_{-\mathbf{k}}, f_{\mathbf{k}}\right]\right] \\
& +\sum_{\substack{\mathbf{0} \neq \mathbf{m} \neq \pm \mathbf{l} \neq \mathbf{0} \\
\mathbf{m}>-\mathbf{m}-\mathbf{l}<\mathbf{l}}} \frac{-1}{(\mathbf{m} \cdot \omega)((\mathbf{m}+\mathbf{l}) \cdot \omega)}\left[f_{\mathbf{m}},\left[f_{\mathbf{l}}, f_{-\mathbf{m}-\mathbf{l}}\right]\right] .
\end{aligned}
$$

In Theorem 5.7, the minus sign in the formula for $\beta_{\mathbf{k}}^{[j]}$ must be deleted.

For the results in Sect. 5.3 to hold, it is necessary to assume that the Hamiltonian functions $I_{j}(x), j=1, \ldots, d$, are in involution.

In formula (78), the minus sign after $=$ must be deleted. 